\title{
Constitutive Modeling of Nonlinear Strain and Time Dependent Behaviors of Ballistic Gelatin at Low Strain Rates
}

\author{
Li LIU*, Chuan DING**, Qianqian LU* \\ *Zhejiang University City College, Hangzhou, 310015, China, E-mail: liulizju@zju.edu.cn \\ **Zhejiang University of Technology, Hangzhou, 310014, China, E-mail: chuanding@zjut.edu.cn \\ cross $^{\text {ref }}$ http://dx.doi.org/10.5755/j01.mech.26.3.22000
}

\section{Introduction}

Ballistic gelatin is a tissue simulant whose properties can be easily modified to match various human organ tissue properties by simply varying water-powder mass ratio. Therefore, it has become a popular model material or surrogate in many bioengineering or biomedical studies [13]. There are several reports on the uniaxial compression, elongation or simple shear experiments on ballistic gelatin [4-10]. The results of these works, including the ones of our group [11, 12], showed highly nonlinear elasticity of ballistic gelatin at large deformation. In addition, the stress-strain behavior of ballistic gelatin is sensitive to the deformation rate, especially at high strain rates, as demonstrated by the Split Hopkinson Pressure Bar (SHPB) experiments published $[5,10]$. Many studies on ballistic gelatin adopted elastic or hyperelastic constitutive models and calibrated the material parameters for each strain rate separately. Among these studies, Amborn et al. [13] adopted three forms of hyperelastic models (Marlow form, NeoHookean form and Ogden form) to describe the nonlinear elastic behaviors of ballistic gelatin. The material parameters were obtained using compression experimental data at a specific strain rate of $300 / \mathrm{s}$ which was considered to be consistent with the application in ballistic penetration. [14] proposed tabulated hyperelasticity to capture strain ratedependence based on numerous actual stress-strain and strain rate curves from experimental tests. However, this method requires a sufficiently rich set of constituent curves to cover the desired range of loading conditions $[15,16]$. [15] employed an Ogden type strain energy density function for the nonlinear elastic response and a single Prony exponential term for the rate-dependent response of ballistic gelatin over multiple strain rates. The calibration of the material parameters was based on uniaxial compression tests with nominal compression strain rates in the range of $0.001 / \mathrm{s} \sim 0.1 / \mathrm{s}$.

In our former work [12], the Mooney-Rivlin model was adopted to describe the large elastic behavior of ballistic gelatin and the material parameters were obtained as functions of the strain rate based on quasi-static compression tests $(\sim 0.208 / \mathrm{s})$ and SHPB tests of [5] ( 1550/s). The drawbacks of this method are that the fitting errors in calibrating of the material parameters are quite large and the application is not convenient due to the need of defining a "strain rate" for any specific problem. Furthermore, the Mooney-Rivlin model cannot describe the nonlinear elasticity of ballistic gelatin in the simple shear tests. In another part of our former work, [11] a linear viscoelastic model, the standard three-element viscoelastic model, is established to describe the rate-dependence of ballistic gelatin at small deformation. This model can predict the shock wave propagations in ballistic gelatin quite well, but it cannot describe the material behaviors under large deformations.

The present study is concerned with the constitutive modeling of ballistic gelatin at quasi-static loading speeds. Uniaxial compression tests and simple shear tests under two modes: relaxation tests at small strain and shear tests at large strain are carried out to study the time and large strain dependent behaviors of ballistic gelatin. A visco-hyperelastic material model, based on the widely used Prony series and reduced polynomial strain energy potential, is proposed for constitutive modeling and the parameters are calibrated using a scheme proposed by [17] and [18]. The material model, with the parameters calibrated by experimental data from our simple shear tests, is then implemented in Abaqus to numerically simulate the uniaxial compression tests and the numerical and experimental results agree well with each other.

\section{Testing procedures}

\subsection{Sample preparation}

One portion of 250 Bloom type-A collagen powder, provided by QingHai Gelatin Factory (China), is mixed with three portions of water at room temperature $\left(18 \sim 25^{\circ} \mathrm{C}\right)$, after 40 minutes, six portions of hot water $\left(70^{\circ} \mathrm{C}\right)$ is added for dissolution to form $10 \mathrm{wt} \%$ gelatin solution. The solution is then sealed and put into a vacuum oven at the constant temperature of $60^{\circ} \mathrm{C}$ and is stirred every 15 minutes. After three times of stirring, the solution is left in the vacuum at a constant temperature of $40^{\circ} \mathrm{C}$ for hydration for 24 hours. Lastly, the solution is poured into a mold to make samples for compression tests or loaded on the plate of a rheometer for simple shear tests and relaxation tests (Fig. 1). In the present study, for each test under the same conditions, we carry it out three times to make sure the results are repeatable.

\subsection{Relaxation tests and results}

Ballistic gelatin solution at $40^{\circ} \mathrm{C}$ is loaded between the parallel plates of a rheomter (Gemini 200), as shown in Fig. 1, a and cooled to $4^{\circ} \mathrm{C}$. The diameter of the upper plate is $40 \mathrm{~mm}$. The gap between the upper rotational plate and the fixed plate is $2 \mathrm{~mm}$. The fixed plate is cooled by a refrigerated circulator (F25-HE, Julabo, temperature resolution is $0.01^{\circ} \mathrm{C}$ ). Low-viscosity silicon oil is coated over the peripheral gap to prevent water evaporation from the sample. The relaxation tests are performed at 
a constant shear strain rate of $0.079 / \mathrm{s}$ up to a strain of 0.05 , then the deformation is held constant thereafter for about 2000 s. A typical strain history is shown in Fig. 2. Only a small fraction of the history, time from $0 \mathrm{~s}$ to $2 \mathrm{~s}$, is displayed here for a clear observation of the ramp rising part. The corresponding shear stress response is shown in Fig. 3. The time axial is displayed in $\log 10$ scale. This set of relaxation test is used later to calibrate the time-dependent parameters of ballistic gelatin.

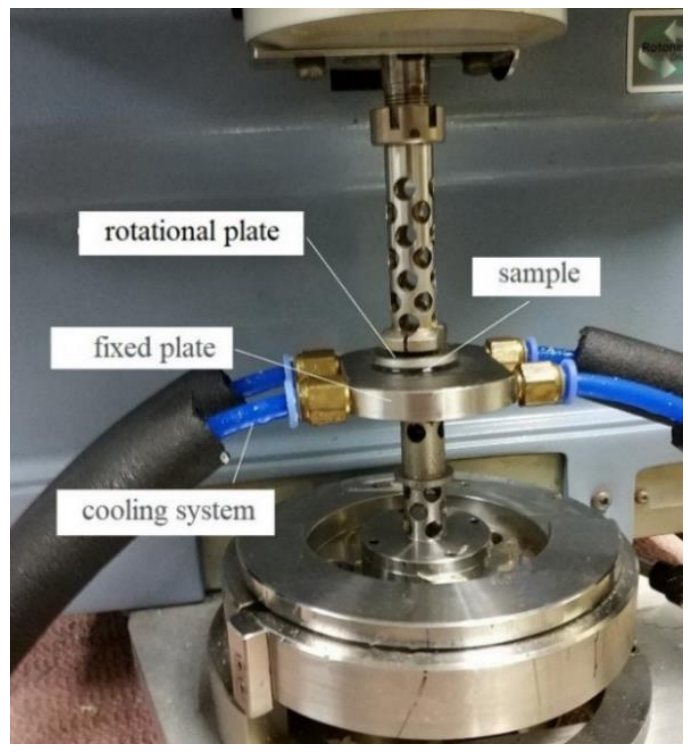

a

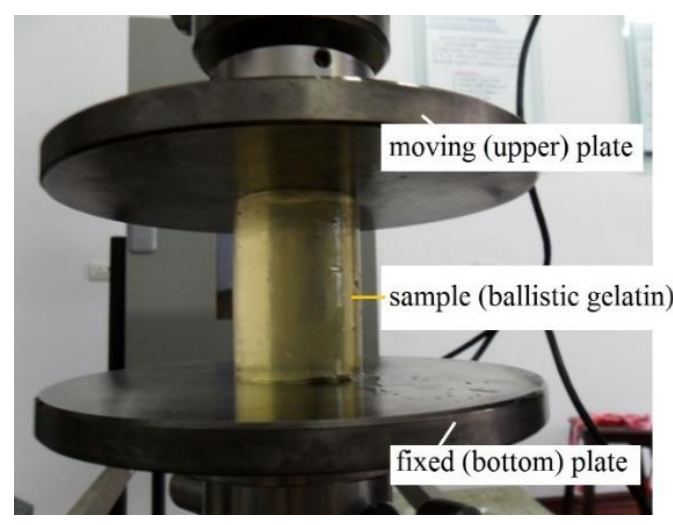

b

Fig. 1 The test configurations: a-simple shear test, b - uniaxial compression test

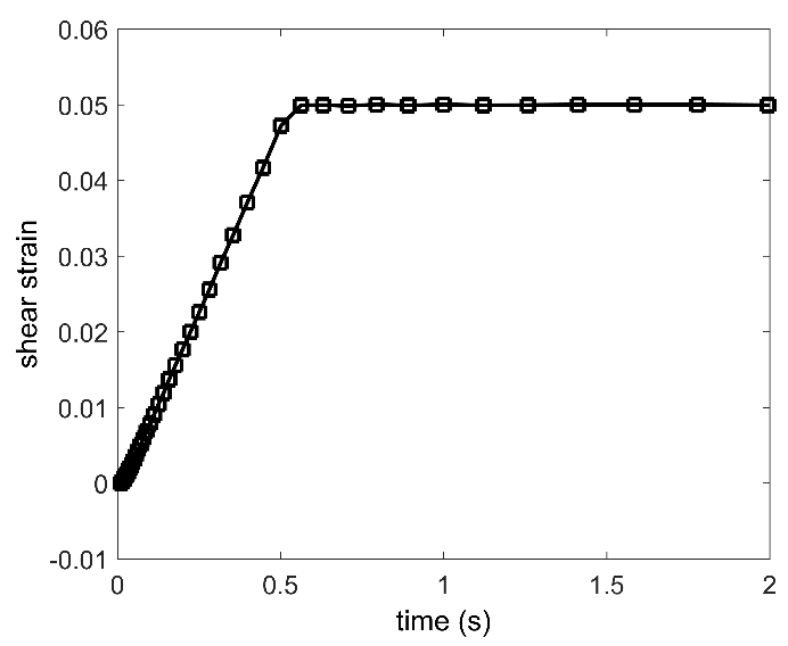

Fig. 2 A typical loading history in the relaxation tests

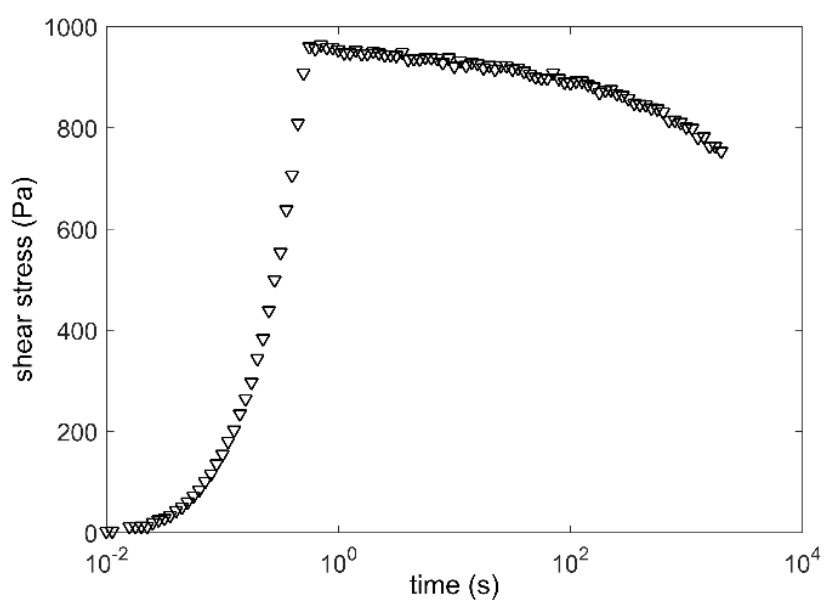

Fig. 3 The stress history in the relaxation test with the loading condition shown in Fig. 2

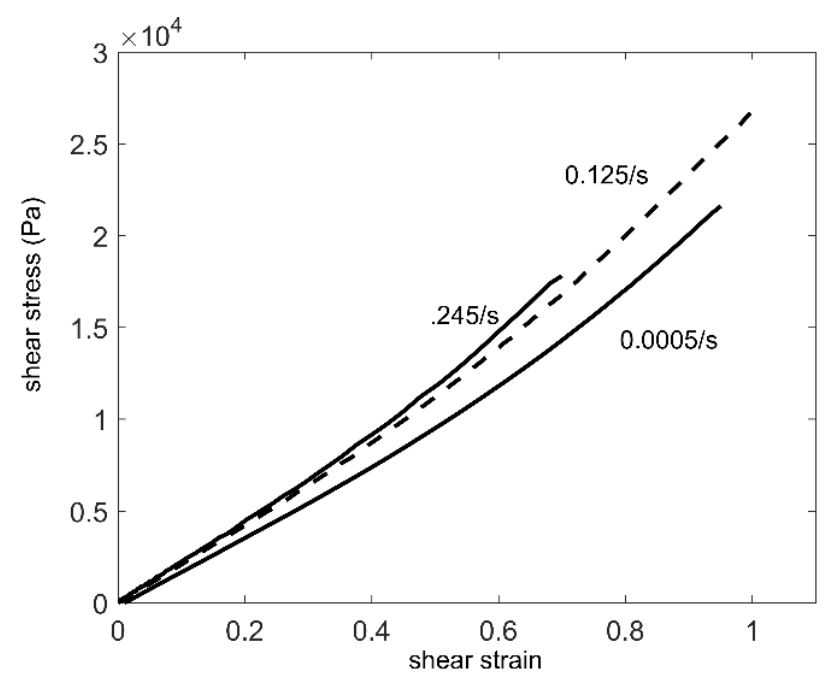

Fig. 4 Shear stress-strain curves in simple shear tests under different shear rates

\subsection{Simple shear and compression tests}

The simple shear tests are performed with the same configuration in Fig. 1, a. The mode of constant shear-strain-rate is adopted and all samples are deformed to shear strains as large as $0.7 \sim 1$ to examine the large elastic behaviors of ballistic gelatin. The stress-strain curve at strain rate of $0.125 / \mathrm{s}$, shown in Fig. 4 , is used later to calibrate the nonlinear strain dependent material model. Then, based on the calibrated material parameters, our material model is used to predict the shear stress-strain curves in the simple shear tests under strain rates of $0.0005 / \mathrm{s}$ and $1.245 / \mathrm{s}$ to validate our material model.

Uniaxial compression tests are carried out on the universal material tester of Zwick/Roell Z020 [12]. The samples (diameter $d=40 \mathrm{~mm}$, length $l_{0}=40 \mathrm{~mm}$ ) are released from the molds just before the compression tests and every test is accomplished as soon as possible (within 3 minutes) to prevent temperature rise. Pure water is used as lubricant on the loading surfaces. Each sample is compressed up to $60 \sim 75 \%$ engineering strain under a constant compression velocity: $10,50,200$ or $500 \mathrm{~mm} / \mathrm{min}$. The true compression stress vs. stretch ratio curves for each compression velocity is shown in Fig. 5. The strain rates in the compression tests are nominal compression strain rates defined as $V / l_{0}$, in which $V$ is the compression velocity. 
The stretch ratio is defined as $\left(1-\Delta l / l_{0}\right)$, in which $\Delta l$ is the compressed length of the sample. The true compression stress is calculated by supposing that the sample is incompressible: $F \cdot(1-\Delta l / l) / A_{0}$, in which $F$ is the compression force and $A_{0}$ is the original cross section area of the sample.

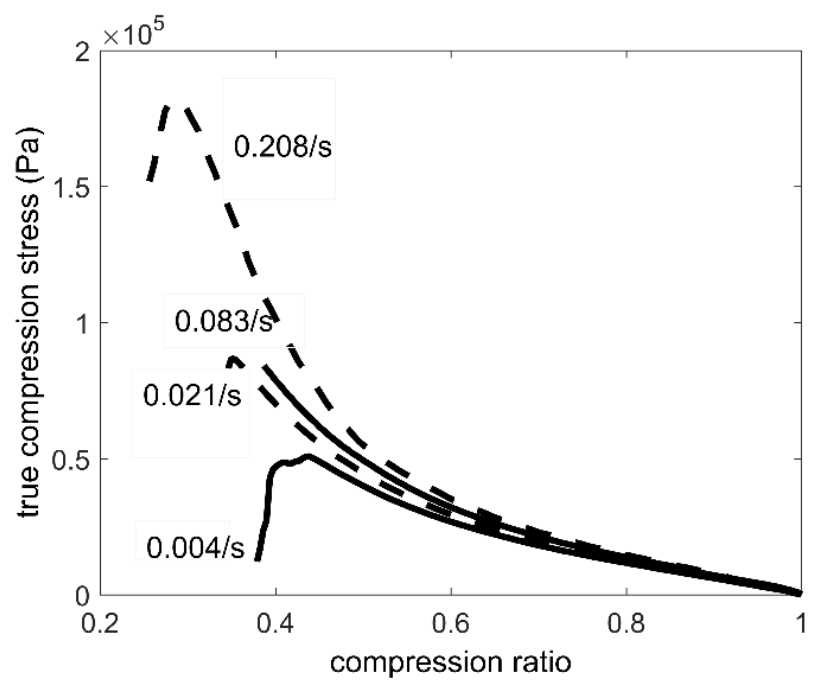

Fig. 5 The stress-strain curves of ballistic gelatin obtained from uniaxial compression tests under different compression rates

\section{Constitutive modelling}

The ballistic gelatin is assumed to be homogeneous, isotropic and incompressible. It is also assumed, and will be justified later, that the time and strain dependent material behaviors are separable. Then the shear stress as a function of the time and the shear strain at a constant temperature can be expressed as:

$$
\sigma_{\tau}=f(\gamma) g(t)
$$

where: $t$ is the time and $f(\gamma)$ and $g(t)$ are the strain and the time dependent functions, respectively. There are various expressions for defining $f(\gamma)$ and $g(t)$. In the present work, the time dependent behavior is defined by the Prony series, which is:

$$
g(t)=g_{\infty}+\sum_{i=1}^{N} g_{i} \exp \left(\frac{-t}{\tau_{i}}\right),\left(g_{\infty}+\sum_{i=1}^{N} g_{i}=1\right)
$$

where: $\tau_{i}(i=1,2,3, \ldots)$ are the time constants. $g_{i}$ and $g_{\infty}$ are dimensionless parameters. The instantaneous strain dependent behavior $f(\gamma)$ can be derived by a widely used strain energy potential as follows [19]:

$$
U=\sum_{i=1}^{N} C_{i 0}\left(\bar{I}_{1}-3\right)^{i}+\sum_{i=1}^{N} \frac{1}{D_{i}}\left(J^{e l}-1\right)^{2 i},
$$

in which $U$ is the strain energy per unit of reference volume; $C_{i 0}$ and $D_{i}$ are material parameters; $\bar{I}_{1}=\bar{\lambda}_{1}^{2}+\bar{\lambda}_{2}^{2}+$ $\bar{\lambda}_{3}^{2}$ is the first deviatoric strain invariant and $\lambda_{i}$ are the deviatoric stretch ratios in principle directions; $J^{e l}$ is the elas- tic volume ratio. In the present study, we adopt $N=2$ and suppose the material is incompressible, so that Eq. (3) is reduced to:

$$
U=C_{10}\left(I_{1}-3\right)+C_{20}\left(I_{1}-3\right)^{2}
$$

in which $I_{1}=\lambda_{1}^{2}+\lambda_{2}^{2}+\lambda_{3}^{2}$ is the first strain invariant of the Finger strain tensor, B. Then the stress can be expressed as follows [20]:

$$
\boldsymbol{\sigma}=-p \mathbf{I}+2 \frac{\partial U}{\partial I_{1}} \mathbf{B}
$$

Combining Eq. (4) and Eq. (5) we obtain:

$$
\boldsymbol{\sigma}=-p \mathbf{I}+2\left[C_{10}+2 C_{20}\left(I_{1}-3\right)\right] \mathbf{B} \text {. }
$$

For simple shear deformation, the Finger tensor is $\mathrm{B}=\left[\begin{array}{ccc}1+\gamma^{2} & \gamma & 0 \\ \gamma & 1 & 0 \\ 0 & 0 & 1\end{array}\right]$ and the first strain invariant is $I_{1}=3+\gamma^{2}$, then

$\boldsymbol{\sigma}=\left[\begin{array}{ccc}-p & 0 & 0 \\ 0 & -p & 0 \\ 0 & 0 & -p\end{array}\right]+2\left(C_{10}+2 C_{20} \gamma^{2}\right)\left[\begin{array}{ccc}1+\gamma^{2} & \gamma & 0 \\ \gamma & 1 & 0 \\ 0 & 0 & 1\end{array}\right]$.

So the shear stress is:

$$
f(\gamma)=2\left(C_{10} \gamma+2 C_{20} \gamma^{3}\right)
$$

If the deformation is small, the higher order term in the above formula can be ignored. Thus $2 C_{10}$ equals the shear modulus $G$ of the material.

Consider a shear test at small strain in which a time varying shear strain, $\gamma(t)$, is applied to the material. The shear stress response, $\sigma_{\tau}(t)$ is given by the convolution integral as follows [21]:

$$
\sigma_{\tau}(t)=\int_{0}^{t} g(t-s) \frac{d f(\gamma(s))}{d s} d s .
$$

In a practical relaxation test, a ramp deformation history (Fig. 2) is applied because of difficulties in imposing a step strain. If the loading up stage is performed at a constant shear strain rate, $\dot{\gamma}_{0}$, then $\gamma(s)=\dot{\gamma}_{0} s$. And Eq. (8) can be solved for the loading up stage using the limits $s=0$ to $s=t$, and the relaxation stage using the limits $s=0$ to $s=t_{0}$, in which $t_{0}$ is the time when the required strain is reached. Fitting the solutions of Eqs. (2), (7) and (8) to the experimental data of our relaxation tests in Fig. 3 yields the material constants in $f(\gamma)$ and $g(t)$. In the expression of $g(t)$ in Eq. (2), the time constants, $\tau_{i}$, are chosen to differ by a factor of 10 within the total duration of the relaxation test. For the present study, four time constants are used: $\tau_{1}=1$, $\tau_{2}=10, \tau_{3}=100, \tau_{4}=1000$. In the first step, the solutions to the convolution integral, supposing $C_{20}=0$ in Eq. (7) since the strain $\gamma$ is very small in the relaxation tests, are 
matched with the test data in Fig. 3 to obtain the values of $C_{10}$ and $g_{i}(i=1,2,3,4)$. In the second step, the strain dependent parameters $C_{20}$ is fitted with the shear stress vs. strain data under large deformation, in Fig. 4, using the fitted parameters $C_{10}$ and $g_{i}(i=1,2,3,4)$ from the first step.

\section{Results and discussions}

The calibrations of the polynomial and Prony series are performed using the Fmincon function in matlab (R2010b), to find a constrained minimum of a scalar function (i.e. the objective function) of several variables (i.e. the material parameters) starting at an initial estimation. The objective function of this problem returns the sum of the squares of the differences between the experimental data and the solutions of Eq. (2), (7) and (8). The calibration result of the model is shown in Fig. 6 and the optimized results of $g_{i}(i=1,2,3,4)$ and $C_{10}$ are listed in Table 1 . The calibration of the polynomial constant, $C_{20}$ in Eq. (7) using the simple shear test results at $0.125 / \mathrm{s}$ is shown in Fig. 7 and the results of $C_{20}$ is also listed in Table 1.

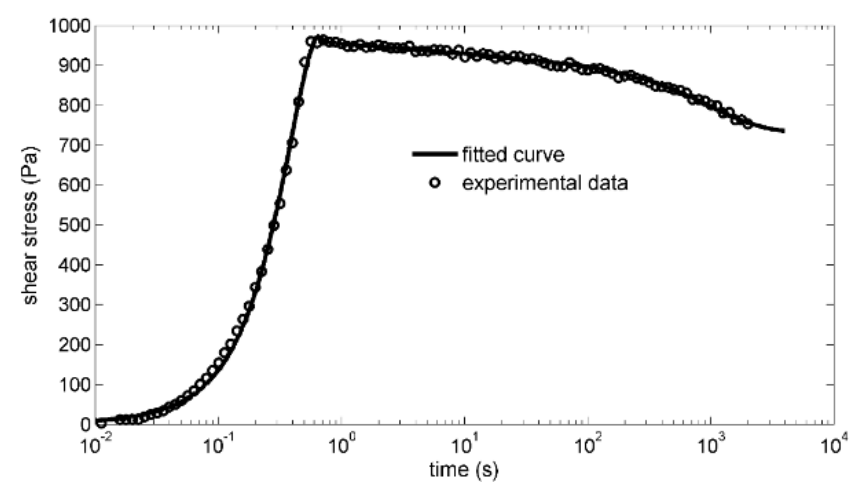

Fig. 6 The fitting result using Prony series in Eq. (2) and polynomial function in Eq. (7). The points are experimental data from the relaxation test

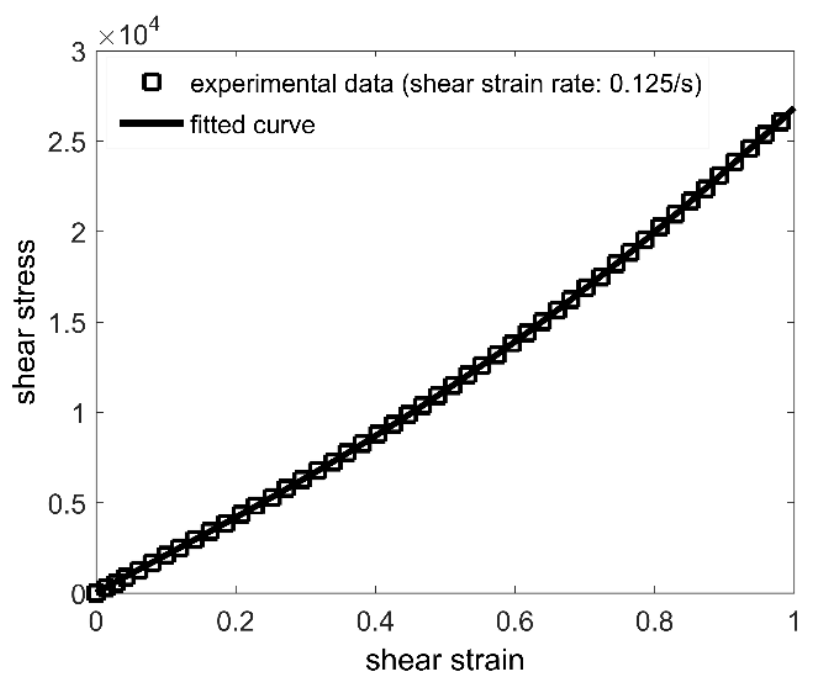

Fig. 7 The fitting result of polynomial function in Eq. (7) using Prony series in Table 1 . The points are experimental data from the simple shear test

With the calibrated parameters of $g_{i}(i=1,2,3,4)$, $C_{10}$ and $C_{20}$ in Table 1, our material model can predict the shear stress-strain relationship for simple shear test at strain rates of $0.0005 / \mathrm{s}$ and $1.245 / \mathrm{s}$ very well as shown in Fig. 8.
Table 1

The calibration results of $g_{i}(i=1,2,3,4)$ by fitting to the relaxation test as shown in Fig. 6

\begin{tabular}{|l|l|l|l|l|l|l|}
\hline$C_{10}$ & $C_{20}$ & $g_{1}$ & $g_{2}$ & $g_{3}$ & $g_{4}$ & $g_{\infty}$ \\
\hline $9.2 \mathrm{e} 3$ & $2.05 \mathrm{e} 3$ & 0.022 & 0.0137 & 0.0449 & 0.202 & 0.717 \\
\hline
\end{tabular}

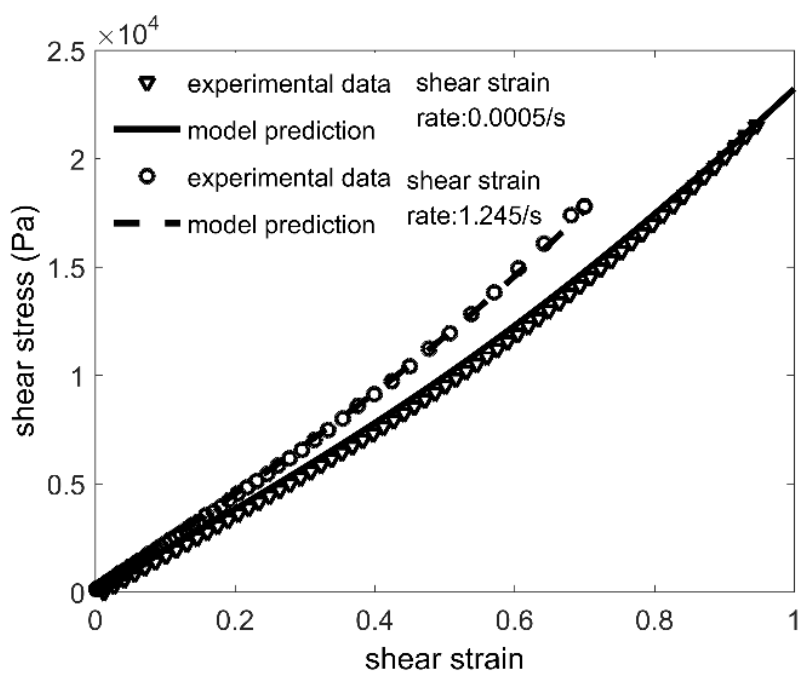

Fig. 8 The comparisons of the model predictions using material model in Eq. (2) and (4) and the simple shear experimental results

The visco-hyperelastic model based on the 4-term Prony series and reduced polynomial strain energy potential (see Eq. (2) and (4)) have been calibrated and validated using the shear test configuration: relaxation at small strain and simple shear at large strain under different strain rates from $0.0005 / \mathrm{s}$ to $1.245 / \mathrm{s}$.

To further verify the material model for the compression tests, the model is implemented in the commercial finite element code of ABAQUS to generate FE solutions for our compression tests under different compression rates, due to the absence of analytical expressions for the stress-strain relationship in the compression experimental configuration. In the FE analysis, the four-node axisymmetric element (CAX4R) is used and the finite element mesh is shown in Fig. 9. The plates are constrained to be rigid bodies to contact and deform the gelatin sample. The interactions between the plates and the sample are defined using the surface to surface contact algorithm. The friction between the plates and the sample is represented by a "penalty" and the friction coefficient is set to be 0.08 , which is the friction coefficient between metal and water [13]. This value is used because ballistic gelatin is mostly composed of water and pure water is used as lubricant between the sample and the plates in our compression tests. The FE solution in ABAQUS generates force-displacement curves of the upper plate under different compression rates and the true compression stress vs. stretch ratio curves can be easily calculated with the same method for the compression experiments.

The comparisons between the model predictions and the experimental data are shown in Fig. 10. Good agreements between the numerical predictions and the experimental data are very encouraging, considering that the material parameters are calibrated by a different type of deformation: simple shear. 


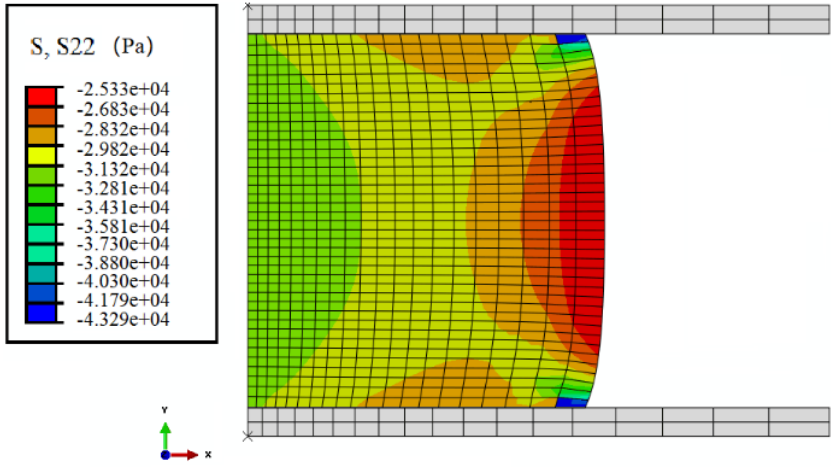

Fig. 9 The FE mesh, deformation and compression stress (Pa) contour at the stretch ratio of 0.656 under the compression rate of $0.208 / \mathrm{s}$

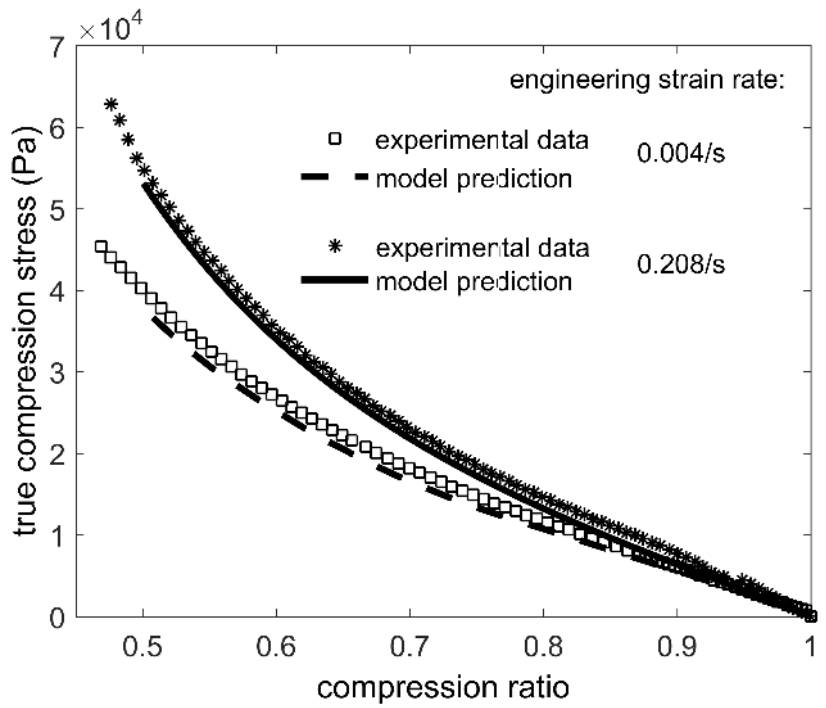

Fig. 10 Comparisons between the numerical model predictions using ABAQUS with material model in Eqs. (2) and (4) and the uniaxial compression experimental results

\section{Conclusions}

The constitutive modeling of ballistic gelatin at quasi-static loading speeds has been studied through experimental and numerical analysis. A visco-hyperelastic material model is proposed to describe the nonlinear strain and time dependent behaviors. The material parameters in the model are calibrated through an indirect approach: the strain dependent behavior is first modeled by a polynomial function and then fitted with the reduced polynomial hyperelastic function; the time dependent behavior is modeled by 5 -term Prony series and calibrated by shear relaxation tests. The calibrated material model can predict the simple shear tests under different strain rates very well. Encouragingly, the numerical stress-strain results predicted by the model with the parameters calibrated from the relaxation and shear tests are in very good agreement with the uniaxial compression experiments, indicating that accurate characterization of the nonlinear strain and time dependent behaviors of ballistic gelatin has been achieved.

\section{Acknowledgements}

This work is supported by the Zhejiang Provincial Natural Science Foundation of China (LQ19A020005 and

\section{LQ19E050018).}

\section{References}

1. Pirlot, M.; Dyckmans, G.; Bastin, I. 2001. Soap and Gelatine for simulating human body tissue: an experimental and numerical evaluation, 19th International Symposium of Ballistics, 7-11 May 2001, Interlaken, Switzerland, Vulnerability Modeling \& Wound Ballistics: 1011-1017.

2. Grover, C. N.; Cameron, R. E.; Best, S. M. 2012, Investigating the morphological, mechanical and degradation properties of scaffolds comprising collagen, gelatin and elastin for use in soft tissue engineering, Journal of the mechanical behavior of biomedical materials 10: 62-74. https://doi.org/10.1016/j.jmbbm.2012.02.028.

3. Jussila, J. 2004. Preparing ballistic gelatine-review and proposal for a standard method, Forensic science international 141(2): 91-98.

4. Moy, P.; Gunnarsson, C. A.; Weerasooriya, T. 2009. Tensile deformation and fracture of ballistic gelatin as a function of loading rate [C], SEM Annual Conference, Albuquerque, NM: Society for Experimental Mechanics Inc.: 848-855.

5. Salisbury, C. P.; Cronin, D. S. 2009. Mechanical properties of ballistic gelatin at high deformation rates, Experimental mechanics 49(6): 829-840.

6. Cronin, D. S.; Falzon, C. 2011. Characterization of $10 \backslash \%$ ballistic gelatin to evaluate temperature, aging and strain rate effects, Experimental mechanics 51(7): 1197--1206. https://doi.org/10.1007/s11340-010-9438-z.

7. Koene, L.; Barou, J. L.; Viot, P. 2011. Ballistic gelatine behaviour under quasi-static and dynamic loadings, in 26th International Symposium On Ballistics. Miami.

8. Kwon, J., et al. 2011. An optical technique for determination of rheological properties of gelatin, Journal of Rheology 55(5): 951-964.

9. Moy, P., et al. 2011. Loading rate effect on tensile failure behavior of gelatins under mode I, in Dynamic Behavior of Materials 1: 15-23.

10. Richler, D.; Rittel, D. 2014. On the testing of the dynamic mechanical properties of soft gelatins, Experimental Mechanics 54(5): 805-815.

11. Liu, L.; Fan, Y.R.; Li, W. 2014. Viscoelastic shock wave in ballistic gelatin behind soft body armor. Journal of the mechanical behavior of biomedical materials 34: 199-207. https://doi.org/10..1016/j.jmbbm.2014.02.011.

12. Liu, L., et al. 2014. A spherical cavity expansion model of large elastic deformation and its application to ballistic gelatin penetration problems, International Journal of Impact Engineering 71: 106-116. https://doi.org/10.1016/j.ijimpeng.2014.04.007.

13. Amborn, E. K.; Muci, K. U.; Chler, K. H.; Hinz, B. J. 2012. Experimental and numerical study of soft tissue surrogate behavior under ballistic loading, American Society of Mechanical Engineers: 743-752.

14. Cronin, D. S. 2011. Ballistic gelatin characterization and constitutive modeling, in Dynamic Behavior of Materials 1: 51-55. 
15. Ravikumar, N., et al. 2015. A constitutive model for ballistic gelatin at surgical strain rates, Journal of the mechanical behavior of biomedical materials 47: 87-94. https://doi.org/10.1016/j.jmbbm.2015.03.011.

16. Kolling, S., et al. 2007. A tabulated formulation of hyperelasticity with rate effects and damage, Computational Mechanics 40(5): 885. https://doi.org/10.1007/s00466-006-0150-x.

17. Goh, S. M.; Charalambides, M. N.; Williams, J. G. 2003. Large strain time dependent behavior of cheese, Journal of Rheology 47(3): 701-716. https://doi.org/10.1122/1.1562153.

18. Goh, S. M.; Charalambides, M. N.; Williams, J. G. 2004. Determination of the constitutive constants of non-linear viscoelastic materials, Mechanics of TimeDependent Materials 8(3): 255-268. https://doi.org/10.1023/B:MTDM.0000046750.65395.f e.

19. Lin, D. C., et al. 2009. Spherical indentation of soft matter beyond the Hertzian regime: numerical and experimental validation of hyperelastic models, Biomechanics and Modeling in Mechanobiology (8): 345. https://doi.org/10.1007/s10237-008-0139-9.

20. Macosko, C. W. 1994. Rheology: principles, measurements, and applications, New York: WILEY-VCH.

21. Williams, J. G., Stress analysis of polymers. Wiley, New York, 1980.
L. Liu, C. Ding, Q. Lu

\section{CONSTITUTIVE MODELING OF NONLINEAR \\ STRAIN AND TIME DEPENDENT BEHAVIORS OF BALLISTIC GELATIN AT LOW STRAIN RATES}

\section{S u m m a r y}

The present study is concentrated in constitutive modeling of ballistic gelatin at low strain rates. The relaxation tests, simple shear tests at strain rates ranging from $0.0005 / \mathrm{s}$ to $1.245 / \mathrm{s}$ and uniaxial compression tests at engineering strain rates ranging from $0.004 / \mathrm{s}$ to $0.208 / \mathrm{s}$ are carried out, and nonlinear strain and time dependent behaviors of ballistic gelatin are observed. A visco-hyperelastic model is proposed based on the Prony series and the reduced polynomial strain energy potential. The material parameters are obtained by fitting to the data of the relaxation and simple shear tests and validated by predicting the compression stress-strain relationships in the uniaxial compression tests. The nonlinear strain and time dependent behaviors of ballistic gelatin are well captured by the model proposed

Keywords: ballistic gelatin; biological material; constitutive modeling; visc- hyperelastic.

Received November 08, 2018 Accepted June 02, 2020 